\title{
Rhizopus oryzae Retro-Orbital Abscess: Comparison of Spores and Hyphae Antifungal Susceptibility and Clinical Outcome
}

\author{
Adenilza Cristina S. Fonseca1, Ana Beatriz A. Teixeira-Loyola ${ }^{2 *}$, Luzia Lyra1, Ariane F. Busso ${ }^{3}$, \\ Yasunori Muraosa4, Hideaki Taguchi ${ }^{4}$, Maria Luiza Moretti ${ }^{\sharp \#}$, Angélica Z. Schreiber ${ }^{1 \dagger}$ \\ ${ }^{1}$ Clinical Pathology Department, School of Medical Sciences, University of Campinas, Campinas, Brazil \\ ${ }^{2}$ Sapucai Valley University, Pouso Alegre, Brazil \\ ${ }^{3}$ Infectious Diseases Division, School of Medical Sciences, University of Campinas, São Paulo, Brazil \\ ${ }^{4}$ Medical Mycology Research, Chiba University, Chiba, Japan \\ Email: ‘analkmim@hotmail.com, ${ }^{*}$ moretti.luiza@gmail.com, ${ }^{\dagger}$ zaninele@fcm.unicamp.br
}

How to cite this paper: Fonseca, A.C.S., Teixeira-Loyola, A.B.A., Lyra, L., Busso, A.F., Muraosa, Y., Taguchi, H., Moretti, M.L. and Schreiber, A.Z. (2018) Rhizopus oryzae Retro-Orbital Abscess: Comparison of Spores and Hyphae Antifungal Susceptibility and Clinical Outcome. Open Journal of Medical Microbiology, 8, 1-11. https://doi.org/10.4236/ojmm.2018.81001

Received: January 20, 2017

Accepted: March 24, 2018

Published: March 27, 2018

Copyright (c) 2018 by authors and Scientific Research Publishing Inc. This work is licensed under the Creative Commons Attribution International License (CC BY 4.0).

http://creativecommons.org/licenses/by/4.0/

\begin{abstract}
Rhizopus is fungus of the order Mucorales, responsible for invasive diseases called mucormycosis. The correct identification of these fungi is one of the main questions raised in the literature since the diagnosis is not simple, being the necessary detection in biopsies of infected tissues and isolation of the microorganism for identification by morphological and molecular methods. In vitro susceptibility testing results are not fully clear since there are reports showing that in vitro resistance does not always mean therapeutic failure. This paper describes a case of rhino-orbito-cerebral mucormycosis in a patient with alcoholic chronic pancreatitis and secondary mellitus diabetes involving microbiological diagnostic, antifungal susceptibility tests for conidia and hypha and clinical correlation. The causative agent was identified by morphological characteristics and DNA sequencing. Minimal Inhibitory Concentration (MIC) for amphotericin B (AMB), itraconazole (ITZ) and terbinafine (TERB) was determined by broth microdilution method. Standardization of the dynamic growth evaluation patterns using the automated system BCT (BioCell-Tracer ${ }^{\circledR}$ ) was obtained allowing to determining hyphae growth control and evaluating the effect of different concentrations of amphotericin B, itraconazole and terbinafine directly on growing hyphae. The fractional inhibitory concentration (FIC) for the combination of the two antifungals that the patient received at the same time, terbinafine and itraconazole, was also determined trying to correlate with clinical outcome. Strains isolated of retro-orbital abscess were identified as Rhizopus oryzae. MIC results (MIC) testing conidia showed high sensibility to amphotericin B and itraconazole in comparison to the obtained for terbinafine. The tests in the automated system
\end{abstract}


BCT using hyphae showed sensibility to all tested antifungal agents and the FIC results for combination in vitro between terbinafine and itraconazole showed synergism consistent with the therapeutic success obtained for the patient who received initial treatment with amphotericin B followed for maintenance treatment with the combination itraconazole and terbinafine

\section{Keywords}

Antifungal Susceptibility Testing, Mucorales, Terbinafine, Amphotericin B, Itraconazole

\section{Introduction}

Mucormycosis is an invasive and difficult to treat opportunistic infection, caused by fungi of the order Mucorales, with poor prognosis in imunocompromissed patients. It occurs mainly in patients with hematologic malignancies, transplant recipients, diabetics, neutropenics or in steroid therapy. Rhizopus oryzae is the main causative agent of mucormycosis, whereas entomophthorales Mucor, Rhizomucor and Cunninghamella bertholletiae are less found. Mucormycosis describes infections characterized by one or more of a triad of rhinocerebral, pulmonary and disseminated disease [1]. The standard therapy for invasive mucormycosis includes reversion of the underlying predisposing factors (if possible), wide-spread surgical debridement of the infected area and adjunctive antifungal therapy, generally with amphotericin B. Many times high doses are required, with limited therapeutic activity and frequently high nephrotoxicity, being not effective particularly in disseminated infections [2]. The diagnosis mucormycosis is not simple and can be confirmed by biopsy of affected tissues, when accessible, and isolation of the causative agent for identification by morphological and molecular methods [1]. The epidemiology and immunopathogenesis of mucormycosis are poorly understood [3] being important the antifungal susceptibility testing to guide treatment associated to the correct identification of the etiologic agent, since different genus and species have different behaviors against antifungals agents. Performing antifungal susceptibility tests enables the identification of therapeutic failures by establishing the microbiological or clinical resistance and also allows the evaluation of therapeutic. Antifungal susceptibility testing by broth microdilution method for Rhizopus oryzae and other filamentous fungi have been established by CLSI M 38 A2 [4], no endpoint were defined. Several reports have shown that resistance in vitro does not always mean therapeutic failure [5]. Questions remain about whether the spore form would be suitable for the antifungal assay whereas in infected tissue, hyphae are the predominant forms [6]. Some studies using other species of fungal hyphae from showed that this form can respond to lower concentrations of antifungal agents than conidia. The determination of MIC (Minimal Inhibitory Concentrations) for moulds could be more reliable with utilization of a method capable to 
quantify the inhibition of hyphal growth in vitro in real time. This quantification permits to mimic a growth situation similar to what happens in the human body [7]-[12]. For the analysis of hyphae growth, it is necessary to standardize the experiment for each microorganism genus considering its particular growth characteristics. The Biocell-tracer ${ }^{\circledR}$ (BCT) method proposes the analysis of the stage of emergence of hyphae and all subsequent stages to determine the antifungal activity, together with records and charts that are automatically generated for better evaluation [7]-[12]. There are few articles in the literature on dynamic assessment of fungal growth and its rate of growth inhibition in touch with antifungal agents and none available with Mucorales [6] [8] [9] [10] [11] [12]. This article describes a case of rhino-orbito-cerebral mucoralomycoses due to Rhizopus oryzae in a patient presenting alcoholic chronic pancreatitis and secondary mellitus diabetes, diagnosed by morphological and molecular methods, antifungal susceptibility testing with conidia and hyphae, interaction obtained by the combination of antifungal agents and clinical correlation of laboratory results and clinical outcome.

\section{Case Report}

A 39-year-old man was hospitalized in the Hospital \& Clinics of the University of Campinas, São Paulo, Brazil in January 2011, with a severe pain in the left ocular movement, decreased vision and edema. Patients' previous conditions were diabetes mellitus type II and chronic alcoholism. Culture from the retroorbital abscess revealed filamentous fungi morphologically identified as Rhizomucor sp. The left orbit was enucleated and patient was treated with AmB deoxycholate for 14 days, liposomal AmB for 7 days followed by Itra $300 \mathrm{mg} / \mathrm{day}$ plus Terb $250 \mathrm{mg} /$ day until April 2012. In the follow-up visit in May 2014 the patient did not present any sign of reactivation of the fungal infection.

The strains isolated from retro-orbital abscess were identified as Rhizomucor sp. The isolates that were inoculated in Sabouraud dextrose agar for $48 \mathrm{~h}$ at $25^{\circ} \mathrm{C}$ showed fast-growing colonies. The colonies appeared a fluffy growth with cotton candy texture, initially white-gray turning dark brown as they became old, while the reverse were bright yellow [13]. Microscopic observation showed irregularly branched rhizoids located at points on the stolon between the sporangiophores and spherical sporangia around $60-100 \mu \mathrm{m}$ in diameter. Molecular identification was performed by sequencing the D1/D2 domain region of the constituent ribosomal 28S DNA using the primer pair: forward NL1 (5'-GCATATCAATAAGC GGAGGAAAAG-3') and reverse NL4 (5'-GGTCCGTGTTTCAAGA CGG-3'). The strain was cultured in potato dextrose agar (Difco) at $28^{\circ} \mathrm{C}$ for 2 days and the DNA was extracted from mycelium using High Pure PCR Template Preparation kit (Roche Applied Science, Germany), according to the manufacturer's specifications. Sequencing reactions were performed using the Big Dye ${ }^{\mathrm{TM}} \mathrm{Ter}-$ minator v.3.1 Kit (Applied Biosystems) in a thermocycler Veriti 96 Well Thermal Cycler (Applied Biosystems). The forward and reverse sequences were aligned in ATSQ program (Software Japan Inc., Japan) and the homology with other sequences was assessed using BLAST (http://blast.ncbi.nlm.nih.gov) and 
Biolomics (http://www.cbs.knaw.nl/). The DNA sequences obtained in our laboratory were identical to the sequences obtained in the Medical Mycology Research Center-Chiba/Japan. The isolate was classified as Rhizopus oryzae with $100 \%$ similarity in the following databases accessed on February 18, 2014.: BLAST (Basic Local Aligment Search Tool, available in http://blast.ncbi.nlm.nih.gov/Blast.cgi) and Biolomics (http://www.cbs.knaw.nl/).

We performed in vitro susceptibility testing to $\mathrm{AmB}$, Terb and Itra, by broth microdilution method [5] with a final inoculum of $4.0 \times 10^{4}$ spores $/ \mathrm{mL}$.

Broth microdilution combination test with Terb and Itra was performed using the "chessboard" methodology to determine the fractional inhibitory concentration (FIC) and the interaction between the antifungal agents (synergism (FIC $\leq$ $0.5)$; indifference $(0.5<\mathrm{FIC} \leq 4)$ and antagonism $(\mathrm{FIC}>4)[10]$.

The monitoring of hyphae growth inhibition was performed in the BioCellTrace $^{\circledR}$ system (BCT) (Hidan Co.,Chiba, Japan) that can monitor the growth rate change of a single hypha at every $30 \mathrm{~s}$ to a precision of $0.1 \mu \mathrm{m} \mathrm{min}^{-1}$ [7] [8]. One $\mu \mathrm{L}$ containing $10^{5}$ spores was dropped in the centre of a culture vessel, coated with $0.02 \%(\mathrm{~W} / \mathrm{V})$ poly-L-lysine (Sigma Chemical), and kept for $60 \mathrm{~min}$ at room temperature. After the incubation, the culture vessel was gently washed with 250 $\mu \mathrm{L}$ of sterile RPMI 1640. Two mL of RPMI were added to the culture vessel and incubated for 18 to $24 \mathrm{~h}$ at $35^{\circ} \mathrm{C}$. After the last incubation, BCT started to monitor the selected hyphae that grew horizontally at the vessel bottom. Growth rate was measured every $3 \mathrm{~min}$.

Spore MICs concentrations for AmB, Itra and Terb alone and Itra and Terb combination were determined by broth microdilution method (CLSI). Spore MICs concentrations for AmB, Itra and Terb alone and the combination of serum concentrations of Itra $(0.15$ a $0.35 \mu \mathrm{g} / \mathrm{mL})$ and Terb (around $1.0 \mu \mathrm{g} / \mathrm{m}$ ) were also analyzed in BCT. The time of experiment was 0 - 15 minutes for Pré-exposure period and after addition of the antifungal agents, Exposure period was extended as much as possible to permit observation of some effect especially when working with the very low serum concentrations.

The spore MIC value of $\mathrm{AmB}$ determined by the broth dilution method was $0.25 \mu \mathrm{g} / \mathrm{mL}$ with $92.57 \%$ hyphae growth inhibitory ratio determined by BCT. For Itra the MIC value was $1.0 \mu \mathrm{g} / \mathrm{mL}$ that promoted no hypha growth inhibition on BCT. Spore MIC value for Terb was $\geq 128 \mu \mathrm{g} / \mathrm{mL}$ and the hyphae growth inhibitory ratio determined by BCT for $128 \mu \mathrm{g} / \mathrm{mL}$ was $98.70 \%$.

Combination in vitro between terbinafine and itraconazole using the "chessboard" methodology [14] showed synergism with FIC $=0.3$. The hyphae growth inhibitory ratio for the serum concentrations of Itra combined with Terb was $49.3 \%$ (Figure 3 ) and could be observed only after 90 minutes of experiment.

\section{Materials and Methods}

\subsection{Microorganism Isolation and Identification}

Sample of retro-orbital abscess was collected and cultures were performed in 
Sabouraud Dextrose agar slants (Difco, Sparks, Maryland, USA) at room temperature. The genus was identified by the classical method that involves phenotypic identification based mainly on morphological characteristics [15].

The species level identification was performed by sequencing the D1/D2 domain region of the constituent ribosomal 28S DNA using the following primer pair: forward NL1 (5'-GCATATCAATAAGCGGAGGAAAAG-3') and reverse NL4 (5'-GGTCCGTGTTTCAAGACGG-3') at the Laboratory of Molecular Epidemiology, FCM-UNICAMP [16]. The strain was cultured in potato dextrose agar (Difco, Sparks, Maryland, USA) at $28^{\circ} \mathrm{C}$ for 2 days and the DNA was extracted from mycelium using the kit High Pure PCR Template Preparation (Roche Applied Science, Germany) according to the manufacturer's specifications. Sequencing reactions were performed using the Big Dye ${ }^{\mathrm{TM}}$ Terminator v.3.1 Kit (Applied Biosystems, Foster City, CA, USA) in a thermocycler Veriti 96 Well Thermal Cycler (Applied Biosystems, Foster City, CA, USA). The forward and reverse sequences obtained were aligned in ATSQ program (Software Japan Inc., Japan) and the homology with other sequences was assessed using BLAST (http://www.westerdijkinstitute.nl/) and Biolomics.

\subsection{Preparation for Monitoring by BioCell-Tracer System}

Plastic tissue culture dishes $\left(35 \times 10 \mathrm{~mm}\right.$, Corning ${ }^{\circledR}$ CellBIND, NY, USA $)$ were used as culture vessels. The conidial cells from 3-day cultures maintained in potato dextrose agar slants (PDA) at $25^{\circ} \mathrm{C}$ were harvested with sterile saline water. The resulting mixture of conidia and hyphae was transferred to a sterile tube and was vortexed for $1 \mathrm{~min}$. Heavy suspension particles were allowed to settle for 3 $5 \mathrm{~min}$. and the upper homogeneous suspension was collected for inoculum adjustment to $1 \times 10^{5}$ conidia/ml by use of Neubauer haemocytometer. One $\mu \mathrm{L}\left(10^{5}\right.$ conidia) of the suspension were dropped into the centre of a culture vessel bottom coated with $0.02 \%$ (W/V) poly-L-lysine (Sigma Chemical Co., Ltd., St. Louis, Mo., U.S.A). The vessel was kept for $60 \mathrm{~min}$ at room temperature then gently washed with $250 \mu \mathrm{L}$ of sterile RPMI 1640. By this procedure, conidia not adhered to the poly-L-lysine were washed out. After addition of $2 \mathrm{ml}$ of RPMI into the culture vessel, it was incubated for 18 to $24 \mathrm{~h}$ at $35^{\circ} \mathrm{C}$. Then, an appropriate hypha that was growing horizontally at the vessel bottom was selected and monitored by the BioCell-Trace ${ }^{\circledR}$ system (Hidan Co., Ltd., Chiba, Japan). BCT ${ }^{\circledR}$ can detect the growth rate change of a single hypha at every $30 \mathrm{~s}$ to a precision of $0.1 \mu \mathrm{m} \mathrm{min}^{-1}$. Its growth rate was measured for about 3 - $3 \mathrm{~min}$. The time of experiment was $0-15$ minutes for Pré-exposure period and 60 minutes for Exposure period.

\section{Results}

Strains isolated of retro-orbital abscess were identified as Rhizomucor sp. by fast-growing colony, spreading all the dishes in $48 \mathrm{~h}$ at $25^{\circ} \mathrm{C}$ on SDA. The colonies appeared fluffy growth with texture of cotton candy, initially white-gray and becoming dark brown with age, while the reverse were bright yellow [14]. By 
microscopic observation irregularly branched rhizoids were found a), located at points on the stolon between the sporangiophores b) and spherical sporangia that were around $60-100 \mu \mathrm{m} \mathrm{c}$ ) in diameter (Figure 1).

After DNA sequencing with confirmation of the sequences obtained by the Medical Mycology Research Center-Chiba/Japan the isolated microorganism was classified as being Rhizopus oryzae with $100 \%$ similarity in the following databases accessed on February 18, 2014.: BLAST (Basic Local Aligment Search Tool, available in http://blast.ncbi.nlm.nih.gov/Blast.cgi) and Biolomics.

The MIC value of AmB against Rhizopus oryzae determined by the broth dilution method was $0.25 \mu \mathrm{g} / \mathrm{mL}$, although the inhibitory ratio of hyphae growth determined by BioCell-Trace ${ }^{\circledR}$ for this concentration to AmB was $88.25 \%$ during exposure to drugs (Exp-GR). For $0.125 \mu \mathrm{g} / \mathrm{mL}$ the inhibition ratio was $81.61 \%$ (Exp-GR). The MIC value of Itraconazole determined by the broth dilution method was $1.0 \mu \mathrm{g} / \mathrm{mL}$. The inhibitory ratio of the hyphae growth determined by BioCell-Trace ${ }^{\circledR}$ for this concentration to Itraconazole was $12.41 \%$ (Exp-GR). For $0.5 \mu \mathrm{g} / \mathrm{mL}$ the growth inhibition ratio was $0.0 \%$ (Exp-GR). The MIC values of terbinafine determined by the broth dilution method was $>128 \mu \mathrm{g} / \mathrm{mL}$. The inhibitory ratio of the hyphae growth determined by BioCell-Trace ${ }^{\circledR}$ for this concentration to terbinafine was $98.70 \%$ (Exp-GR). For $64 \mu \mathrm{g} / \mathrm{ml}$ the inhibition ratio was $97.44 \%$ (Exp-GR) (Figure 2). The result of the test of the combination in vitro between terbinafine and itraconazole accomplished by using the methodology of the "chessboard" [17] showed synergism. The inhibitory ratio of the hyphae growth determined by BioCell-Trace ${ }^{\circledR}$ for this concentration to terbinafine combined with itraconazole was 49,3\% (Exp-GR) (Figure 3).

\section{Discussion}

The morphological and molecular identifications for this isolate were discordant since it was identified as Rhizomucor sp. by morphology and as Rhizopus oryzae after DNA sequencing. Some studies in the literature have also reported disagreement between these two methodologies showing that the strains usually in disagreement are also regarded as Rhizopus oryzae [13] [17] [19]. Morphologic identification at the genus level is frequently imprecise. Study with 20 mucorales isolates using the automated rep-PCR and ITS sequences as molecular tools

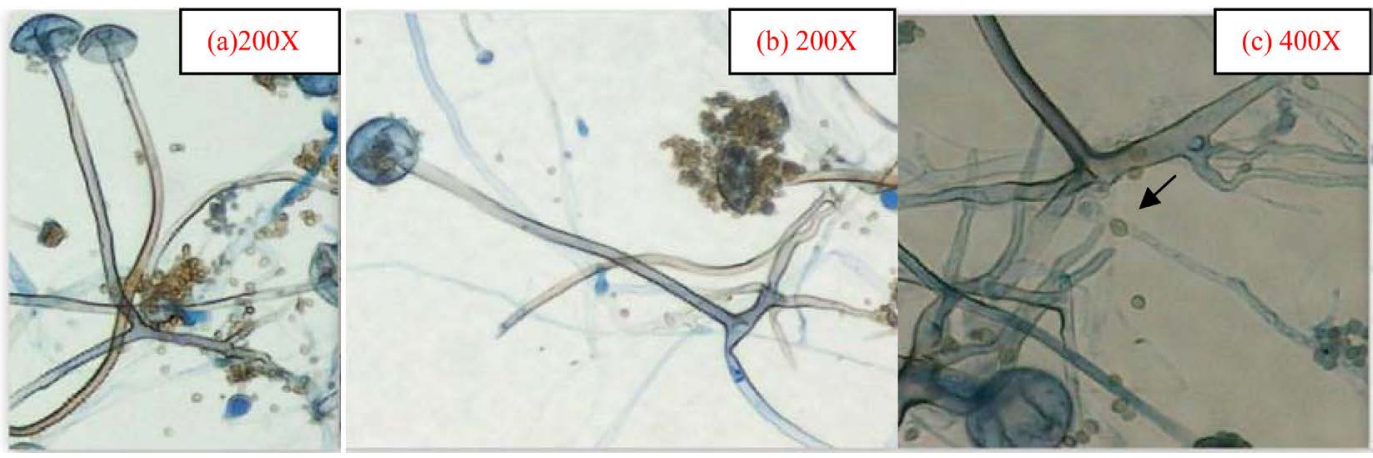

Figure 1. Characteristic structures of Rhizomucor sp. observed with cotton blue lactophenol. 


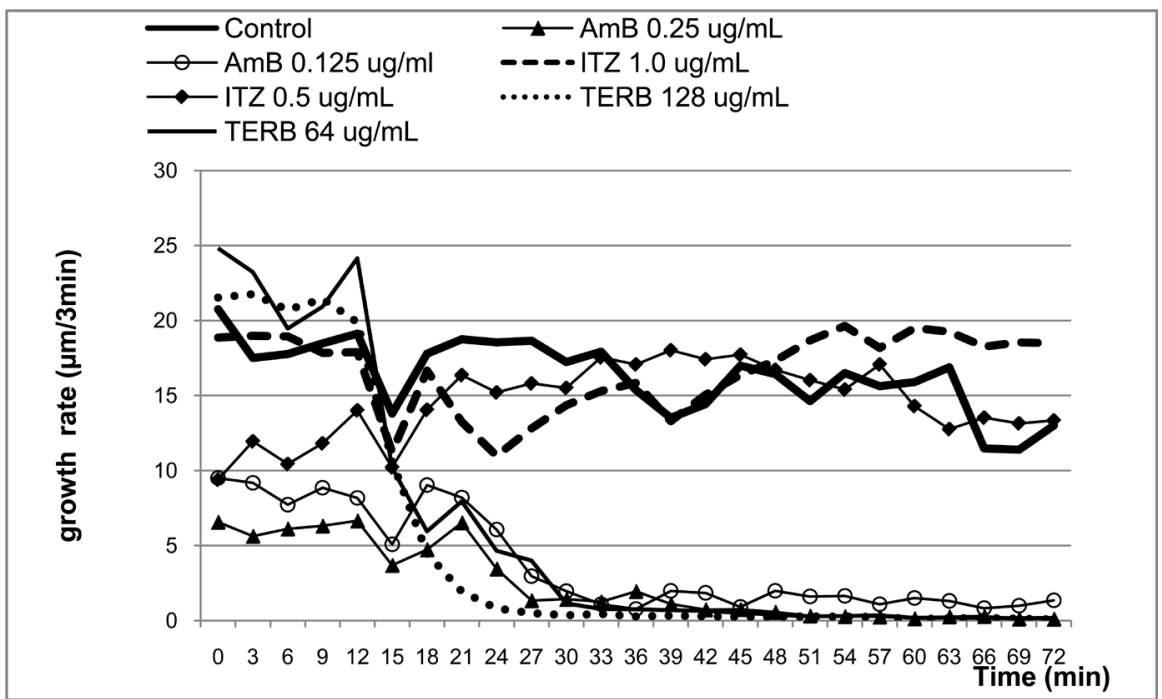

Figure 2. Dynamic response curves of Rhizopus oryzae to various concentrations of amphotericin B (AmB), Itraconazole (ITZ) and Terbinafine (TERB).

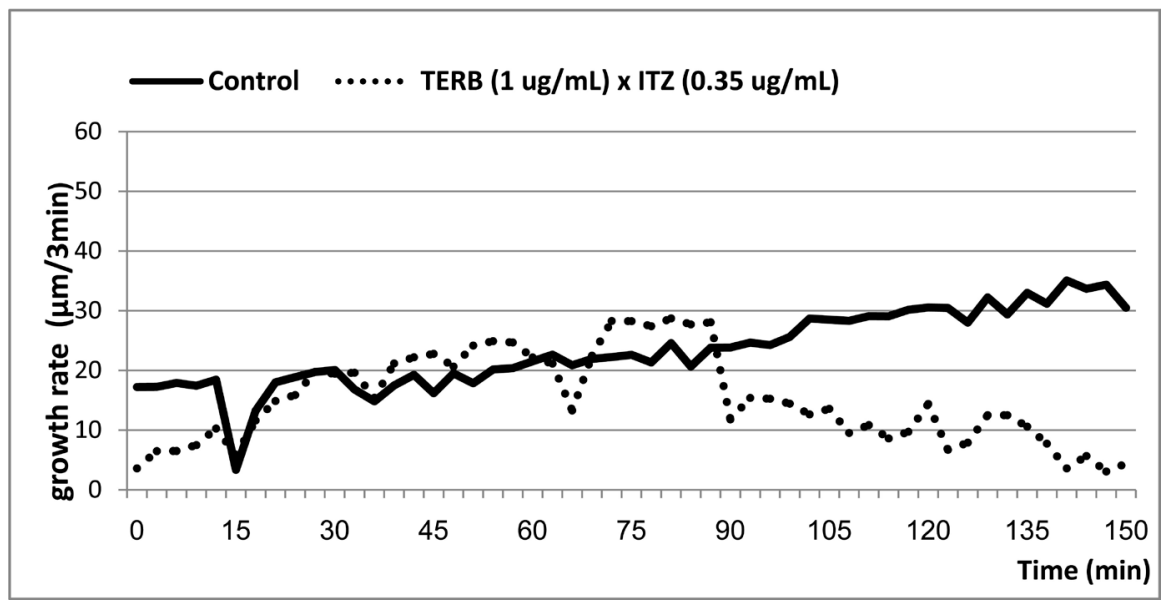

Figure 3. Dynamic response curves of Rhizopus oryzae to combination of Itraconazole (ITZ) and Terbinafine (TERB).

showed $100 \%$ concordance to each other but only $74 \%$ concordance between morphologic and sequence-based identification for the determination of the genus [13]. Many laboratories not identify genus and the species is often omitted in the literature since these consider this difficult task, especially when the observations do not match the descriptions or illustrations of the literature [20]. The application of molecular biology techniques in the identification of mucorales species is complicated by the fact that these are genetically different from most opportunistic fungi. However, the development of new techniques may enable a more accurate diagnosis than the diagnosis obtained with conventional methods [21]. Culture is still the method most used and many published studies do not report the identification of species and some noted a discrepancy in the determination of genus based on morphology and sequencing [19]. Molecular techniques show a great potential to identify quickly and accurately the etiologic 
agents of mucormycosis. However, detection of molecular assays for these fungi are still not widely available [16].

The information available in databases are still limited, there are few data in the literature for this group of micro-organisms. Thus, the need of the more sequences deposited in the databases to enable precise identification of Mucorales may contribute to research on the effectiveness of antifungal since many articles reported differences in the profiles of in vitro susceptibility among species and genus of Mucorales against different antifungal agents. The results of the tests of the minimum inhibitory concentration (MIC) of our study using conidia of strain Rhizopus oryzae showed high sensibility to the amphotericin B and itraconazole in comparison to the terbinafine. The tests in the automated system BCT using hyphae showed sensibility of the strain to the tested antifungal agents and the test of the combination of terbinafine and itraconazole showed synergism being consistency with therapeutic success of the patient which received initial treatment with amphotericin B and then as maintenance treatment the combination of itraconazole and terbinafine. Some articles reported that azole compounds alone or in combination has beneficial effects in animal models infected by Rhizopus sp., and may provide alternatives to the treatment of the mucormycosis [22] [23] [24]. There are no reports in the literature on standardization of automatic growth Biocell-Tracer ${ }^{\circledR}$ to strains of Mucorales isolates. There are few articles in the literature on dynamic assessment of fungal growth and its rate of growth inhibition in touch with antifungal agents. Some studies using hyphae of the other fungal species showed that the shape of hyphae can respond to lower concentrations of the antifungal agents conidia and may have a better correlation with the effect of the antifungal treatment in the infections since the hyphae is the predominant shape in infected tissue. The standardization of the test of assessment dynamic growth for the strain of Rhizopus oryzae in our study showed different results of the standardization for Aspergillus sp. [25] [13] and dermatophytes [12], whereas the mucorales have a behavior completely different from other fungi studied in the literature being necessary several tests and the adaptation of the methodology in our study. That is because the growth of these fungal to be faster being on average of the $9 \mu \mathrm{m} / 3 \mathrm{~min}$ while for dermatophytes this growth ranged from 0.35 to $2.02 \mu \mathrm{m} / 10 \mathrm{~min}, 1.63$ to 8.71 for Aspergillus and Fusarium 1.0 - $6.5 \mu \mathrm{m} / 5 \mathrm{~min}$. Thus more studies of the antifungal susceptibility tests alone or in combination for mucorales are needed to help with the appropriate treatment and standardization of the methodology since the broth microdiluiton method is standardized only for some filamentous fungi such as species of the Fusarium sp., Aspergillus sp., Rhizopus sp., Pseudallescheria boydii and Sporothrix schenkii and each species has a different behavior in the test being important the standardization of the methodology. It is also important to conduct further studies using the method of growth dynamic evaluation what may present a better clinical correlation, whereas the form of hyphae is the morphological form present in tissues of infected individuals. 


\section{Acknowledgements}

We thank CAPES and the Research Center for Pathogenic Fungi and Microbial Toxicosis, Chiba University, Japan. Adenilza Cristina da Silva Fonseca is a master degree student supported by grants from CAPES (Coordenação de Aperfeiçoamento de Pessoal de Nível Superior) - Brazil. We also thank Luzia L. Fernandes for technical support.

\section{Conflicts of Interest}

All authors relate no conflicts of interest.

\section{Funding}

This project was approved by the Ethical Committee of the School of Medical Sciences - University of Campinas [No. 236/2011].

This work was supported by JICA (Japan International Cooperation Agency) and SATREPS (Science and Technology Research Partnership for Sustainable Development) [02P-29548-09].

Student supported by grants from CAPES (Coordenação de Aperfeiçoamento de Pessoal de Nível Superior) - Brazil.

\section{References}

[1] Rogers, T.R. (2008) Treatment of Zygomycosis: Current and New Options. Journal of Antimicrobial Chemotherapy, 61, 35-39. https://doi.org/10.1093/jac/dkm429

[2] Ibrahim, A., Spelberg, B. and Edwards, J. (2008) Iron Acquisition: A Novel Prospective on Mucormycosis Pathogenesis and Treatment. Nacional Institute of Health, 21, 620-625. https://doi.org/10.1097/QCO.0b013e3283165fd1

[3] Chamilos, G., Lewis, R.E., Lianchun, J.H., Xiao, L., Zal, T., Gilliet, M., Halder, G. and Kontoyiannis, P. (2008) Drosophila Melanogaster as a Model Host to Dissect the Immunopathogenesis of Zygomycosis. PNAS, 105, 9367-9372. https://doi.org/10.1073/pnas.0709578105

[4] Clinical and Laboratory Standards Institute (2008) Reference Method for Broth Dilution Antifungal Susceptibility Testing of Filamentous Fungi. 2nd Edition, Approved Standard, CLSI Document M38-A2. 28 (16).

[5] Lass-Flörl, C., Mayr, A., Perkhofer, S., Hinterberger, G., haudorfer, J., Speth, C. and Fille, M. (2008) Activities of Antifungal Agents against Yeasts and Filamentous Fungi: Assessment according to the Methodology of the European Committee on Antimicrobial Susceptibility Testing. Antimicrobial Agentes and Chemotherapy, 52, 3637-3641. https://doi.org/10.1128/AAC.00662-08

[6] Guarro, J., Llop, C., Aguilar, C. and Pujol, I. (1997) Comparison of In Vitro Antifungal Susceptibilities of Conidia and Hyphae of Filamentous Fungi. Antimicrobial Agents and Chemotherapy, 41, 2760-2762.

[7] Oh, K., Yang, H.C., Matsuoka, A. and Kurata, H. (1995) Combined Effect of Amphotericin B and Fluocytosine on Gromth of Candida albicans Estimated at a Single Hypha Level. Journal of Medical and Veterinary Mycology, 33, 1-4.

[8] Taguchi, H., Miyaji, M. and Nishimura, K. (1995) Studied on the Synergistic Effect of Amphoterecin B and 5-Fluorocytosine on the Growth of Single Hyphae of $A s$ - 
pergillus fumigatus by a Biocell-Tracer System. Mycoscience, 36, 341-344. https://doi.org/10.1007/BF02268610

[9] Ansheng, L., Taguchi, H., Miyagi, M., Nishimura, K. and Shaoxi, W. (1999) Study on the Hyphal Responses of Aspergillus fumigatus to the Antifungal Agent by Biocell-Tracer. Mycopathology, 148, 17-23.

[10] Iida, Y., Oh, K., Saito, M., Matsuoka, H., Kurata, H., Natsume, M. and Abe, H. (1999) Detection of Antifungal Activity in Aemarrhena asphodeloides by Sensitive BCT Method and Isolation of Its Active Compound. Journal of Agricultural and Food Chemistry, 47, 584-587. https://doi.org/10.1021/jf980707t

[11] Teixeira, A.B.A., Moretti, M.L. and Schreiber, A.Z. (2005) Evaluation of F. solani Hyphae and Conidia Susceptibility to Amphotericin B and Itraconazole: Study of a Clinical Case. Mycophatologia, 116, 291-296.

https://doi.org/10.1007/s11046-005-0106-2

[12] Biancalana, F.S., Lyra, L., Moretti, M.L., Kamei, K. and Schreiber, A.Z. (2011) Standardization of Hyphal Growth Inhibition Rate as a Means of Evaluating Microsporum spp. In Vitro Susceptibility to Terbinafine, Griseofulvin, and Ciclopiroxolamine. Mycophatologia.

[13] Healy, M., Walton, D., Reece, K., Lising, M., Bittner, T., Frye, S. and Kontoyiannis, D.P. (2004) Species Identification of Clinical Zygomycetes Isolates by Automated rep-PCR and DNA Sequencing. Interscience Conference on Antimicrobial Agents and Chemotherapy 44th General Meeting, Washington DC, 30 October-2 November 2004.

[14] Wang, S.B., Li, R.Y. and Yu, J. (2011) Identification and Susceptibility of Rhizomucor spp. Isolated from Patients with Cutaneous Zygomycosis in China. Medical Mycology, 49, 799-805.

[15] Dannaoui, E. (2009) Molecular Tools for Identification of Zygomycetes and the Diagnosis of Zygomycosis. Clinical Microbiology and Infection, 15, s.5. https://doi.org/10.1111/j.1469-0691.2009.02983.x

[16] Voigt, K., Cigelnik, E. and O’Donnell, K. (1999) Phylogeny and PCR Identification of Clinically Important Zygomycetes Based on Nuclear Ribosomal DANN Sequence Data. Journal of Clinical Microbiology, 37, 3957-3964.

[17] Eliopoulos, G.M. and Mollering Jr., R.C. Antimicrobial Combinations. In: Lorian, V., Ed., Antibiotics in Laboratory Medicine, 3 Edition, Williams \& Wilkins, Baltimore, 432-492.

[18] Chakrabarti, A., Ghosh, A., Prasad, G.S., et al. (2003) Apophysomyces Elegans: Na Emerging Zygomycete in India. Journal of Clinical Microbiology, 41,783-788. https://doi.org/10.1128/JCM.41.2.783-788.2003

[19] Kontoyiannis, D.P., Lionakis, M.S., Lewis, R.E., Chamilos, G., Healy, M., Perego, C., Safdar, A., Kantarjian, H., Champlin, R., Walsh, T.J. and Raad, I.I. (2005) Zygomycosis in a Tertiary-Care Cancer Center in the Era of Aspergillus Active Antifungal Therapy: A Case Control Observational Study of 27 Recent Cases. The Journal of Infectious Diseases, 191, 1350-1360. https://doi.org/10.1086/428780

[20] Scholer, H.J., Muller, E. and Schipper, M.A.A. (1983) Mucorales, In: Howard, D.H., Ed., Fungi Pathogenic for Humans and Animals, Part A. Biology. Marcel Dekker, New York, 9-59.

[21] Kappe, R., Okeke, C.N., Fauser, C., et al. (1998) Molecular Probes for the Detection of Pathogenic Fungi in the Presence of Human Tissue. Journal of Medical Microbiology, 47, $811 \pm 820$.

[22] Sheehan, D.J., Hitchcock, C.A. and Sibley, C.M. (1999) Current and Emerging 
Azole Antifungal Agents. Clinical Microbiology Reviews, 12, 40-79.

[23] Mosquera, J., Warn, P.A., Rodriguez-Tudela, J.L. and Denning, D.W. (2001) Treatment of Absidia corymbifera Infection in Mice with Amphotericin B and Itraconazole. Journal of Antimicrobial Chemotherapy, 48, 583-586.

https://doi.org/10.1093/jac/48.4.583

[24] Sugar, A.M. and Liu, X.P. (2000) Combination Antifungal Therapy in Treatment of Murine Pulmonary Mucormycosis: Roles of Quinolones and Azoles. Antimicrobial Agents and Chemotherapy, 44, 2004-2006.

https://doi.org/10.1128/AAC.44.7.2004-2006.2000

[25] Teixeira, A.B.A. (2006) Evaluation Antifungal Susceptibility by the BioCell-Tracer ${ }^{\circledR}$ System Monitoring Hyphal Growth of Species Aspergillus and Fusarium. Doctoral Thesis, Faculty of Medical Sciences, State University of Campinas. 